\title{
Diffuse Juvenile Bullous Pemphigoid Managed Successfully With a Short Course of Cyclophosphamide
}

\author{
Seema Rani ${ }^{1}$, Diksha Aggarwal ${ }^{1}$, Kabir Sardana ${ }^{1}$, Savitha Bathula ${ }^{1}$, Purnima Malhotra ${ }^{2}$
}

\footnotetext{
1 Department of Dermatology, Atal Bihari Vajpayee Institute of Medical Sciences (ABVIMS) and Dr. Ram Manohar Lohia Hospital, New Delhi, India

2 Department of Pathology, Atal Bihari Vajpayee Institute of Medical Sciences (ABVIMS) and Dr. Ram Manohar Lohia Hospital, New Delhi, India
}

Key words: immunobullous, bullous pemphigoid, cyclophosphamide, juvenile bullous pemphigoid

Citation: Rani S, Aggarwal D, Sardana K, Savitha B, Malhotra P. Diffuse juvenile bullous pemphigoid managed successfully with a short course of cyclophosphamide. Dermatol Pract Concept. 2022;12(1):e2022005. DOI: https://doi.org/10.5826/dpc.1201a05

Accepted: April 18, 2021; Published: January 2022

Copyright: $@ 2022$ Rani et al. This is an open-access article distributed under the terms of the Creative Commons Attribution-NonCommercial License (BY-NC-4.0), https://creativecommons.org/licenses/by-nc/4.0/, which permits unrestricted noncommercial use, distribution, and reproduction in any medium, provided the original authors and source are credited.

Funding: None.

Competing interests: None.

Authorship: All authors have contributed significantly to this publication.

Corresponding author: Seema Rani, Associate Professor, Department of Dermatology, Atal Bihari Vajpayee Institute of Medical Sciences (ABVIMS) and Dr. Ram Manohar Lohia Hospital, New Delhi, India. E-mail: drseemashekhar@gmail.com

\section{Introduction}

Adolescent bullous pemphigoid (BP) is a very rare entitywith generalized (erythrodermic) variant being even rarerand management of such difficult BP cases can be challenging.

\section{Case Presentation}

A 17-year-old girl presented with a 1-month history of intense pruritus that was followed by the development of multiple fluid-filled lesions. These lesions ruptured spontaneously in 3 to 4 days and left behind painful erosions. There was no history of drug intake, trauma, recent vaccination, fever,or photosensitivity. Notably, she had an intellectual disability; however, the psychiatric consultation was normal. Cutaneous examination revealed multiple tensevesicles, both clear and hemorrhagic, and bullae over an erythematous base that involved $90 \%$ of the body surface areaalong with involvement of head and neck areas (Figure1A). Nails and mucosa were unaffected. Bulla spread sign was positive and Nikolsky sign was negative. Tzanck smear showed eosinophils and neutrophils. The total leukocyte count was 20,000 per $\mathrm{mm}^{3}$ with $77 \%$ eosinophils in differential leukocyte count and an absolute eosinophil count of 15,400 per $\mathrm{mm}^{3}$. The serum immunoglobulin (Ig) E level was normal. Histopathology revealed focal clefting at the dermoepidermal junction and perivascular mononuclear inflammatory infiltrate with numerous eosinophils in the superficial dermis (Figure 1B). Direct immunofluorescence revealed linear deposits of IgG and C3 along the basement membrane zone. Based on clinical presentation and histopathological and immunological findings, a diagnosis of diffuse adolescent BP was made. The BP disease area index score was 145 . 


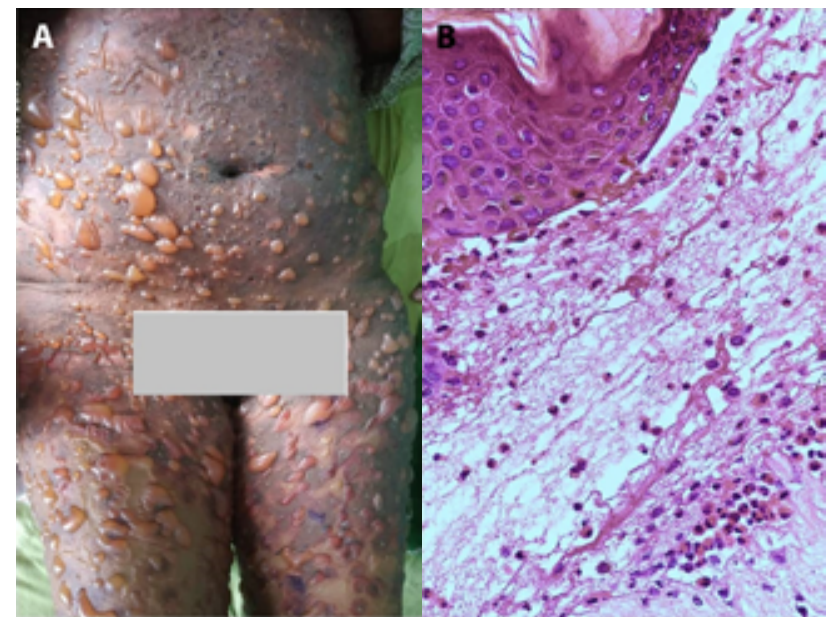

Figure 1. (A) Multiple tense vesicles and bullae over an erythematous base. (B)Subepidermal blister containing fibrin and eosinophils peri adnexal infiltrate of eosinophils in the dermis (hematoxylin and eosin stain, $\times 40$ ).

Oral prednisolone $70 \mathrm{mg}$ along with broad-spectrum antibiotic cover and antihistamines were started. Doxycycline $100 \mathrm{mg}$ and nicotinamide tablets $500 \mathrm{mg}$ were added as steroid-sparing agents, but there was a progressive increase in the severity of the eruption. As a consequence, Doxycycline was replaced by azathioprine $50 \mathrm{mg}$ twice daily. In spite of 4 weeks of azathioprine, there were 40-50 new blisters per day, and in view of the recalcitrance, azathioprine was discontinued and cyclophosphamide $100 \mathrm{mg}$ was added. After 2 weeks, the number of new lesions gradually decreased and old lesions started to heal. The patient was discharged on prednisolone $50 \mathrm{mg}$ and cyclophosphamide $100 \mathrm{mg}$. Two weeks later the dose of cyclophosphamide and prednisolone was tapered to $50 \mathrm{mg}$ and $40 \mathrm{mg}$, respectively. During treatment patient routine hematological investigations and urineanalysis were normal. Over the next 4 weeks, cyclophosphamide was stopped. Azathioprine was restarted at a dose of $25 \mathrm{mg}$ and gradually increased. Currently the patient is undergoing oral prednisolone $15 \mathrm{mg}$ and azathioprine $50 \mathrm{mg}$ with a good control of her disease without any side-effects. The patient is on regular follow-up at a 4- to 6-week intervals with no relapse of bullous lesions but with residual pigmentary changes (Figure 2).

Informed consent has been taken from patient guardian (patient is minor) for the nature and side-effects of drug (cyclophosphamide).

\section{Conclusions}

$\mathrm{BP}$ is the commonest immunobullous disorder worldwide, but it is rare in childhood (100 cases) and extremely uncommon in adolescence (14 cases) [1]. Childhood BP is diagnosed according to the criteria of Nemeth, and our case satisfied these criteria. BP is generally more responsive to treatment than pemphigus vulgaris, but there are refractory cases in

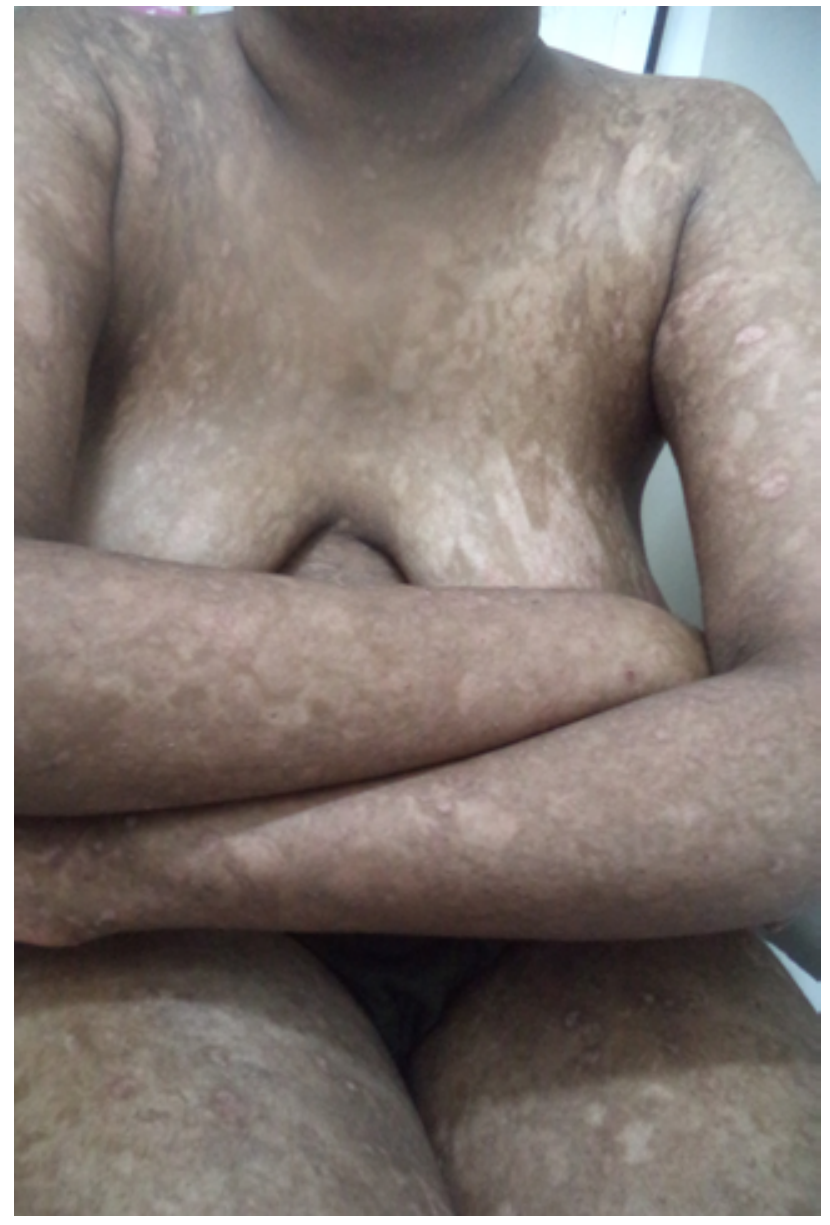

Figure 2. Healed lesions with residual pigmentary changes.

which treatment resistance occurs. Childhood BP usually has a good prognosis, although, in some cases the course is less benign [2]. Our case had generalized BP, poor response to high doses of steroid, anti-inflammatory, antibiotics and azathioprine. Non-availability of biologics at our center and economic constraints resulted in the use of cyclophosphamide that was administered as a "rescue therapy" in view of its side effect profile. Our case exemplified the role of cyclophosphamide as a short-term bridge therapy, after which the patient was adequately controlled on azathioprine. In a severe and recalcitrant case, such as this one, cyclophosphamide can be used to bring the disease under control.

Informed consent: Written informed consent for publication of clinical details and clinical images was obtained from the patient.

\section{References}

1. Patsatsi A, Kyriakou A, Werth VP. Bullous pemphigoid in adolescence. Pediatr Dermatol. 2019;36(2):184-188. DOI: 10.1111/ pde.13717. PMID: 30569520.

2. Nemeth AJ, Klein AD, Gould EW, Schachner LA. Childhood Bullous Pemphigoid: Clinical and Immunologic Features, Treatment, and Prognosis. Arch Dermatol. 1991;127(3):378-386. doi:10.1001/archderm.1991.01680030098014. PMID: 1998369. 